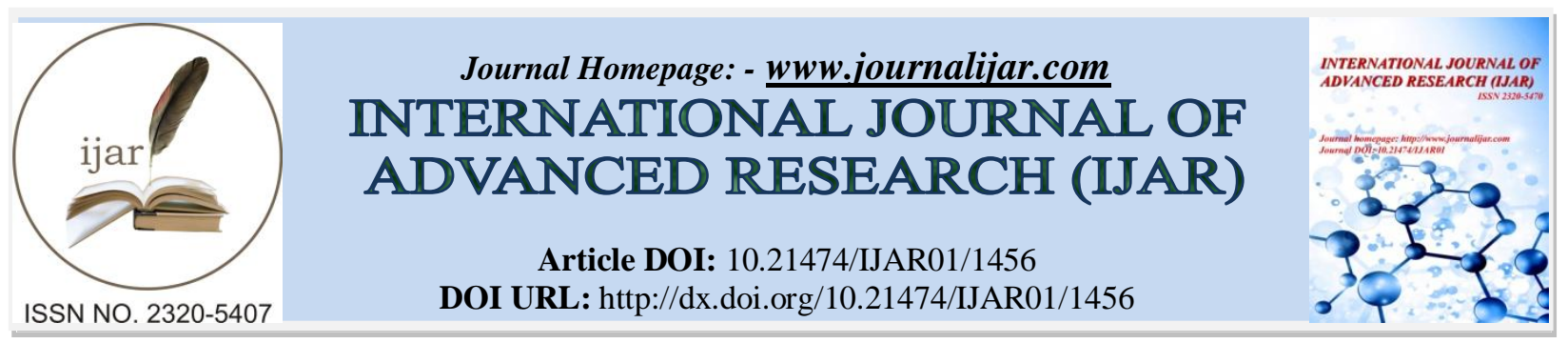

RESEARCH ARTICLE

\title{
UNIQUENESS OF FRACTIONAL DIFFERENTIAL EQUATIONS USING GRONWALL TYPE INEQUALITIES.
}

\section{G. U. Pawar ${ }^{1}$ and J. N. Salunke ${ }^{2}$.}

1. Department of Mathematics, Mahatma Jyotiba Fule Jr. College,Pishor-431104,Dist.Aurangadad (M.S.), India.

2. School of Mathematical Sciences, Swami Ramanand Teerth Marathwada University, Nanded (M.S.), India.

\section{Manuscript Info}

Manuscript History

Received: 19 July 2016

Final Accepted: 15 August 2016

Published: September 2016

Key words:-

Initial value problem (IVP),

Generalized Gronwall -type inequalities,

Boundedness and uniqueness.

\section{Abstract}

In this paper we study the boundedness and uniqueness of solution of initial value problem by using Gronwall type integral inequalities.

\section{Introduction:-}

Fractional calculus deals with the investigations of derivatives and integrals of any arbitrary real or complex order ( non-integer order ). The fractional order differentiation and integration represents a rapidly growing field both in theory and applications of real world problems. The class of fractional differential equations of various types plays important roles in physics, control systems, dynamical systems and engineering, porous media [ 1,3,7 ] [5].In the recent years, there has been a significant development in fractional calculus and fractional differential equations $[1,5,8]$ Different aspects of solutions of differential equations can be resolved by using differential and integral inequalities. Perhaps Gronwall type inequalities and their generalizations is the most useable tool in the literature. The integral inequalities involving functions of one and more than one independent variables which provide explicit bounds on unknown functions play a fundamental role in the development of the theory of differential equations [16]. As an application of Generalized Gronwall -type inequalities, we prove the uniqueness and boundedness of solutions of the R-L fractional differential equation of order $0<\alpha<1$.

In this paper we consider the following initial value problem

$$
\begin{aligned}
& \qquad \mathrm{D}_{x}^{\alpha} u(x)=f(x, u(x)), 0 \leq x \leq X . \\
& \text { with initial condition } \\
& \left.\mathrm{D}_{x}^{\alpha-1} u(x)\right|_{x=0}=\delta
\end{aligned}
$$

Where $0<\alpha<1, f \in C(R \times R, R)$, $\mathrm{D}_{x}^{\alpha}$ denotes the Riemann-Liouville fractional derivative defined by

$$
\mathrm{D}_{x}^{\alpha} v(x)=\frac{1}{\Gamma(1-\alpha)} \frac{d}{d x} \int_{0}^{x}(x-t)^{-\alpha} v(t) d t
$$

In this paper, we apply some new generalized Gronwall-type inequalities suitable for the qualitative and quantitative analysis of the solutions to fractional differential equations. In section 2 we introduce some preliminary definitions and results. In section 3 we investigate a certain fractional differential equation, in which the boundedness and 
uniqueness on initial data for the solution to the fractional differential equation are investigated by the use of the generalized Gronwall-type inequalities.

\section{Preliminaries:-}

The definitions of Riemann-Liouville fractional derivative and integral are given in [5].

Definition(2.1)[5]: Let $f \in L_{1}[a, b], \alpha \in \mathbb{R}^{+}$, the Riemann - Liouville fractional integral of order $\alpha$ is defined as

$$
\mathrm{I}_{x}^{\alpha} v(x)=\frac{1}{\Gamma(\alpha)} \int_{a}^{x}(x-s)^{-\alpha} v(s) d s \quad \text { for all } x \in[a, b] .
$$

The author in [4] studied IVP (1.1)-(1.2) for existence and uniqueness. The equivalent integral equation corresponding to IVP(1.1)-(1.2) is

$$
u(x)=\frac{\delta}{\Gamma(\alpha)} x^{\alpha-1}+\frac{1}{\Gamma(\alpha)} \int_{0}^{x}(x-t)^{\alpha-1} f(t, u(t)) d t
$$

Now we present some generalized Gronwall-type inequalities studied by [4] which are required to prove the boundedness and uniqueness of IVP (1.1)-(1.2).

Lemma (2.1)[2]: Assume that $a \geq 0, p \geq q \geq 0$ with $p \neq 0$. Then, for any $K>0$, we have

$$
a^{\frac{p}{q}} \leq \frac{q}{p} K^{\frac{q-p}{p}} a+\frac{p-q}{p} K^{\frac{q}{p}}
$$

Theorem(2.2)[2]: Suppose that $\alpha>0, p \geq 1$ are constants, $L \epsilon C\left(R_{+} \times R_{+}, R_{+}\right)$with

$$
0 \leq L(t, u)-L(t, v) \leq T(u-v) \quad \text { for } u \geq v \geq 0,
$$

where $T$ is the Lipschitz constant $u, a, h$ are nonnegative functions locally integrable on $[0, X)$ with $h$ nondecreasing and bounded by $M$, where $M$ is a positive constant. If the following inequality is satisfied:

$$
u^{p}(x)=a(x)+\frac{1}{\Gamma(\alpha)} h(x) \int_{0}^{x}(x-t)^{\alpha-1} L(t, u(t)) d t, 0 \leq x<X .
$$

then we have the following explicit estimate for $u$ :

$$
\begin{gathered}
u(x) \leq\left\{\tilde{a}(x)+\int_{0}^{x}\left[\sum_{n=1}^{\infty}\left(\frac{T}{P} K^{\frac{1-p}{p}}\right)^{n} \frac{h^{n}(x)}{\Gamma(n \alpha)}(x-t)^{n \alpha-1} \tilde{a}(t)\right] d t\right\}^{\frac{1}{p}} \\
\text { Where } \tilde{a}(x)=a(x)+\frac{1}{\Gamma(\alpha)} h(x) \text { and } K>0 \text { is a constant. }
\end{gathered}
$$

\section{Main Result:-}

We prove boundedness and uniqueness of solution of IVP (1.1)-(1.2) in this section.

Theorem(3.1): For IVP( ), if $|f(x, u)| \leq L(x,|u|)$, where $L$ is defined as in the Theorem(2.2), then we have the following estimate :

$$
u(x) \leq \frac{x^{\alpha-1}}{\Gamma(\alpha)}|\delta|+\left[\sum_{n=1}^{\infty}\left(\frac{T}{P} K^{\frac{-2}{3}}\right)^{n} \frac{x^{(n+1) \alpha-1}}{\Gamma(n+1) \alpha}|\delta|\right], 0 \leq x<X .
$$

where $K>0$ is a constant, and $T$ is defined as in Theorem(2.1).

Proof: The integral equation corresponding to IVP (1.1)-(1.2) is

$$
u(x)=\frac{\delta}{\Gamma(\alpha)} x^{\alpha-1}+\frac{1}{\Gamma(\alpha)} \int_{0}^{x}(x-t)^{\alpha-1} f(t, u(t)) d t
$$

Therefore,

$$
\begin{aligned}
|u(x)| & \leq\left|\frac{\delta}{\Gamma(\alpha)} x^{\alpha-1}\right|+\frac{1}{\Gamma(\alpha)} \int_{0}^{x}(x-t)^{\alpha-1}|f(t, u(t))| d t \\
& \leq \frac{x^{\alpha-1}}{\Gamma(\alpha)}|\delta|+\frac{1}{\Gamma(\alpha)} \int_{0}^{x}(x-t)^{\alpha-1} L(t,|u(t)|) d t, 0 \leq x<X .
\end{aligned}
$$


Then application of Theorem (2.2) gives

$$
\begin{aligned}
u(x) & \leq \frac{x^{\alpha-1}}{\Gamma(\alpha)}|\delta|+\int_{0}^{x}\left[\sum_{n=1}^{\infty}\left(\frac{T}{P} K^{\frac{-2}{3}}\right)^{n} \frac{(x-t)^{n \alpha-1}}{\Gamma(n \alpha)} \frac{t^{\alpha-1}}{\Gamma(\alpha)}|\delta|\right] d t \\
& =\frac{x^{\alpha-1}}{\Gamma(\alpha)}|\delta|+\left[\sum_{n=1}^{\infty}\left(\frac{T}{3} K^{\frac{-2}{3}}\right)^{n} \frac{x^{(n+1) \alpha-1} B(\alpha, n \alpha)}{\Gamma(n \alpha) \Gamma(\alpha)}|\delta|\right] \\
& =\frac{x^{\alpha-1}}{\Gamma(\alpha)}|\delta|+\left[\sum_{n=1}^{\infty}\left(\frac{T}{3} K^{\frac{-2}{3}}\right)^{n} \frac{x^{(n+1) \alpha-1}}{\Gamma(n+1) \alpha}|\delta|\right], \quad 0 \leq x<X .
\end{aligned}
$$

which is the required result.

Theorem(3.2): If $|f(x, u)-f(x, v)| \leq L(x,|u-v|)$, where $L$ is defined as in the

Theorem (2.2) , and $L(t, 0) \equiv 0$, then IVP (1.1) - (1.2) has a unique solution.

Proof: Suppose that IVP (1.1)-(1.2) has two solutions $u_{1}(x)$ and $u_{2}(x)$.

Then we have

$$
\begin{aligned}
& u_{1}(x) \frac{\delta}{\Gamma(\alpha)} x^{\alpha-1}+\frac{1}{\Gamma(\alpha)} \int_{0}^{x}(x-t)^{\alpha-1} f\left(t, u_{1}(t)\right) d t \\
& u_{2}(x) \frac{\delta}{\Gamma(\alpha)} x^{\alpha-1}+\frac{1}{\Gamma(\alpha)} \int_{0}^{x}(x-t)^{\alpha-1} f\left(t, u_{2}(t)\right) d t
\end{aligned}
$$

Now

$$
\begin{aligned}
u_{1}(x)-u_{2}(x) & =\frac{1}{\Gamma(\alpha)} \int_{0}^{x}(x-t)^{\alpha-1}\left[f\left(t, u_{1}(t)\right)-f\left(t, u_{2}(t)\right)\right] d t \\
\left|u_{1}(x)-u_{2}(x)\right| & \leq \frac{1}{\Gamma(\alpha)} \int_{0}^{x}(x-t)^{\alpha-1}\left|f\left(t, u_{1}(t)\right)-f\left(t, u_{2}(t)\right)\right| d t \\
& \leq \frac{1}{\Gamma(\alpha)} \int_{0}^{x}(x-t)^{\alpha-1} L\left(t,\left|u_{1}(t)-u_{2}(t)\right|\right) d t
\end{aligned}
$$

Consider $\left|u_{1}(t)-u_{2}(t)\right|$ as independent function and applying Theorem (2.2) to (3.6), we get

$$
\left|u_{1}(x)-u_{2}(x)\right| \leq 0
$$

This completes the proof.

$$
\text { which implies } u_{1}(x)=u_{2}(x) \text {. }
$$

\section{Conclusions:-}

In this paper, by using generalized inequalities of Gronwall type we establish the boundedness and uniqueness aspects of solution of fractional differential equations. While proving the uniqueness of solution of fractional differential equations integral inequalities proves a powerful technique.

\section{References:-}

1. A.A.Kilbas, H.M.Srivastava and J.J.Trujillo, Theory and Applications of Fractional Differential Equations, Elsevier, Amersterdam,2006.

2. Jiang, F.C., Meng, F.W. : Explicit bounds on some new nonlinear integral inequality with delay. J. Comput. Appl. Math.205, 479-486 (2007).

3. Oldham K.B., Spanier J: The Fractional Calculus, Academic Press,New York(1974).

4. Qinghua Feng and Fanwei Meng, Some new Gronwall-type inequalities arising in the research of fractional differential equation.

5. Podlubny I.,Fractional Differential Equations. Academic Press, San Diego(1999).

6. Pachpatte, BG: Explicit bounds on certain integral inequalities. J. Math. Anal. Appl. 267, 48-61 (2002).

7. Ye, HP, Gao, JM, Ding, YS: A generalized Gronwall inequality and its application to a fractional differential equation. J. Math. Anal. Appl. 328, 1075-1081 (2007)

8. K.S.Miller and B.Ross, An Introduction to the Fractional Calculus and Differential Equations, John Wiley \& Sons, New York, NY, USA,1993.

9. Pachpatte B.G., Inequalities for Differential and Integral Equations, Volume 197 of Mathematics in Science and Engineering, Academic Press Inc, San Diego, CA, 1998. 
10. Pachpatte B.G., Integrals and finite difference inequalities and applications, North Holland Mathematics Studies, 205, Elsevier Science, 2006.

11. Pachpatte B.G., On discrete inequalities related to Gronwall's inequality, Proc. Indian Acad. Sci. Math. Sci. Part A 85 (1977) 26-40.

12. Agrawal R.P., Deng S.F., Zhag W.N., Generalization of a retarded Gronwall-like inequality and its applications, Appl. Math. Comput. 2005, 165,599-612.

13. V. Lakshmikantham, A.S. Vastsala, General uniqueness and monotone iterative technique for fractional differential equations, Appl. Math.Lett. 21 (2008) 828-834.

14. V. Lakshmikantham, A.S. Vastsala, Theory of fractional differential inequalities and applications, Commun. Appl. Anal. 11(july-october) (2007) 395-402.

15. Z.Denton, A.S. Vatsala, Fractional integral inequalities and applications, Computers and Mathematics with Applications 59(2010) 1087-1094. 